\title{
Inborn errors of purine metabolism in man: development of purine enzyme assays using high performance liquid chromatography
}

\author{
H. J. RYLANCE, RUTHC. WALLACE, AND G. NUK I \\ From the Rheumatic Diseases Unit, University Department of Medicine (WGH), Northern General Hospital, Edinburgh \\ EH5 $2 D Q$
}

Several inherited purine enzyme defects have been associated with purine overproduction and gout, nephrolithiasis and immunodeficiency diseases. These include: adenosine deaminase (ADA) deficiency (severe combined immunodeficiency), nucleoside phosphorylase (NP) deficiency (isolated T cell deficiency), nucleotidase (5'NT) deficiency (X-linked and acquired hypogammaglobulinaemia) xanthine oxidase (XO) deficiency (xanthinuria), adenine phosphoribosyl transferase (APRT) deficiency (renal calculi 2, 8-dihydroxy-adenine), h y poxanthine-guanine phosphoribosyl transferase (HGPRT) deficiency (Lesch-Nyhan syndrome or severe X-linked gout) and phosphoribosyl pyrophosphate synthetase (PRPPS) superactivity (X-linked gout with or without nerve deafness and neurodevelopmental abnormalities). Red blood cell enzyme assays have been developed that are rapid and sensitive and avoid the need for radiochemicals in clinical biochemistry laboratories. Haemolysates are prepared from heparinised venous blood samples and assayed for purine enzyme concentrations by methods based on a reverse phase HPLC separation of purine bases, nucleosides, and nucleotides.

H G P R T ${ }^{1}$

Hypoxanthine (HX) + 1 pyrophosphoylribosyl-5-phosphate (PPriboseP) $\rightarrow$ hypoxanthine ribonucleotide (IMP) + inorganic pyrophosphate $\left(\mathrm{PP}_{\mathrm{i}}\right)$.

Incubation mixture contains 0.63 HX, $1.05 \mathrm{mmol}$ PPribose P, $5.3 \mathrm{mmol}$

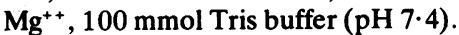

HPLC to separate IMP from HX.

Table 1 Normal values of enzyme activities. Figures are mean (SD)

\begin{tabular}{llc}
\hline HGPRT & $(\mathrm{n}=20)$ & $94(15) \mathrm{nmol} \mathrm{IMP} / \mathrm{mgHb} / \mathrm{hr}$ \\
APRT & $(\mathrm{n}=7)$ & $16(5 \cdot 3) \mathrm{nmol} \mathrm{AMP} / \mathrm{mgHb} / \mathrm{hr}$ \\
PRPPS & $(\mathrm{n}=9)$ & $71 \cdot 2(15 \cdot 7) \mathrm{nmol} \mathrm{PRPP} / \mathrm{mgHb} / \mathrm{hr}$ \\
ADA & $(\mathrm{n}=8)$ & $0 \cdot 28(0 \cdot 07) \mathrm{IU} / \mathrm{ml}$ packed RBC \\
PNP & $(\mathrm{n}=11)$ & $6 \cdot 21(1 \cdot 21) \mathrm{IU} / \mathrm{ml}$ packed RBC
\end{tabular}

Column $\mu$ Bondapack C $18(3.9 \times 300$ $\mathrm{mm})$.

Eluents: $0.02 \mathrm{~mol} / 1$ phosphate buffer (pH 5.6) (A) and methanol/water $(60 \% \mathrm{v} / \mathrm{v})(\mathrm{B})$ Gradient linear $100 \%$ $A$ to $40 \% \mathrm{~B}$ in 35 minutes. Flow rate: $1.5 \mathrm{ml} / \mathrm{min}$. Injection volume $40 \mu \mathrm{L}$.

\section{A P R T}

Adenine (Ad) + PPribose $\mathrm{P} \rightarrow$ adenosine monophosphate (AMP) $+\mathrm{PP}_{\mathrm{i}}$.

Reaction conditions as for HGPRT with substitution of adenine for hypoxanthine. Isocratic HPLC separation of AMP and Ad using phosphate buffer $(\mathrm{pH} 6.0)$ containing $6 \%(\mathrm{v} / \mathrm{v})$ methanol as eluate.

\section{P R P P S}

Ribose-5-phosphate + ATP $\rightarrow$ PPribose P + AMP.

Two stage reaction: $0.05 \mathrm{mmol}$ ribose-5-phosphate + ATP reacted in phosphate buffer $(\mathrm{pH} 7 \cdot 5)$ with $\mathrm{Mg}^{++}$ EDTA and glutathione to form PPriboseP. PPriboseP assayed in HGPRT reaction using excess of partially purified enzyme.

$\mathrm{A} \mathrm{D} \mathrm{A}^{2}$

Adenosine $\rightarrow$ inosine

Haemolysate reacted with $0 \cdot 14$ mmol adenosine in $0.05 \mathrm{mmol}$ phosphate buffer ( $\mathrm{pH} 7 \cdot 5)$.

Isocratic HPLC separation of adenosine using phosphate buffer $(\mathrm{pH}$ $6 \cdot 0)$.

PNP

Inosine $\rightarrow$ hypoxanthine
Haemolysate reacted with $0 \cdot 2 \mathrm{mmol}$ inosine in $0.05 \mathrm{~mol}$ phosphate buffer (pH 7.5).

HPLC as in HGPRT assay.

In patients with gout and hyperuricaemia a sequence of investigations is undertaken:

(1) Serum uric acid + creatinine.

(2) 24-hr urinary uric acid + creatinine + 'purine profile' (normal diet-alcohol, tea, coffee). If $>3.6$ mmol/24 hrs: stage 3 .

(3) Repeat 24-hr urinary uric acid + creatinine + 'purine profile' on 2600 $\mathrm{kcal}, 70 \mathrm{~g}$ protein purine-free diet five days). If $>3.6 \mathrm{mmol} / 24 \mathrm{hrs}$. H Y PE RE X C RET O R OVERPRODUCER.

(4) Measure plasma purines + nucleosides.

(5) Measure RBC levels

PPribose $\mathbf{P}$

PRPPS

HGPRT

APRT

(6) If abnormal-kinetic studies, family studies, heterozygote detection, etc.

\section{References}

1 Rylance H J, Wallace R C, Nuki G. Hypoxanthine guanine phosphoribosyl transferase: assay using high performance liquid chromatography. Clin Chim Acta 1982; 121: 159-65.

2 Hartwick R, Jeffries A, Krstulovic A, Brown P R. An optimised assay for adenosine deaminase using reverse phase high pressure liquid chromatography. $J$ Chromatogr Sci 1978; 16: 427-35. 